\title{
Venezia, Biblioteca Nazionale Marciana, gr. Z. 155 (coll. 610)
}

I.-VI. Costantinopoli, seconda metà del XIV sec.

Comp.; ff. I-II, 1-206, III-IV; sul f. Ir il pinax, tratto dal catalogo di Zanetti (1741), indica il contenuto; sul f. 1r ex libris di Bessarione; num. nel marg. sup. est. a penna: il numero 201 è ripetuto due volte. Sono bianchi i ff. 34v-35r, 194v, 198v. Legatura in cuoio risalente alle campagne effettuate nel 1722, come riportato sul f. IIv.

Il Marc. gr. Z. 155 raccoglie sei unità differenti, riferibili alla seconda metà del XIV secolo. La prima sezione è stata realizzata dopo il 1351, giacché reca il tomo del Sinodo contro Barlaam e Acindino emanato in quest'anno; la terza sezione, con la Parva Refutatio dello ieromonaco Gregorio Acindino, è stata realizzata dopo il 1344, anno in cui Acindino ricevette l'ordinazione (Cañellas 1995, LXXV).

\section{I. ff. 1-16 (Costantinopoli, post 1351)}

Cart.; fil. ff. 1, 4, 7, 8, 11, 12, 14 simile a couteau (M/Tr nr. 3292, 1320); $1^{16}$ (16); num. fasc. assente; mm $276 \times 202$ = 34 // 205 // $35 \times 20$ // 144 // 37; a piena pagina, ll. 26, unità di rigatura mm 6; rigatura assente (f. 11r).

\section{Scrittura}

Nell'unità è presente una sola mano: copista A (ff. 1r-16v), riferibile al XIV secolo.

\section{Contenuto}

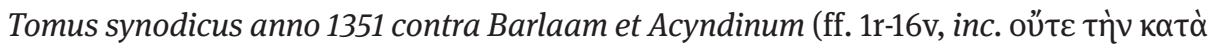

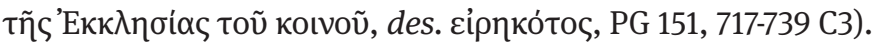

\section{II. ff. 17-34 (Costantinopoli, XIV sec.)}

Cart.; fil. ff. 17, 18, 21, 22 simile a arbalète (M/Tr nr. 1342, 1350), ff. 25, 27, 29, 31 simile a pot (M/Tr nr. 6854, 1341); $2^{8}(24), 3^{8+2}(34)$; num. fasc. presente nel marg. sup. est. del recto del primo e nel marg. inf. int. del verso dell'ultimo foglio di ogni fascicolo, numerati da $\alpha^{\prime}$ a $\beta^{\prime} ; \mathrm{mm} 277 \times 205=30$ // 200 // $44 \times 15$ // 145 // 42; a piena pagina, ll. 35, unità di rigatura mm 7; rigatura assente (f. 32). 


\section{Scrittura}

La sezione è vergata da due copisti anonimi: mano A (f. 17rv) e mano B (ff. 18r-34r). Quest'ultimo fa mostra di due espressioni grafiche, una corsiva (ff. 18r-20r, 1. 2, 22r, 1. 30-24r, 1. 4, 27v-34r, 1. 23) e una posata (ff. 20r, 1.3-22r, 1. 29, 24r, 1. 5-27r).

\section{Decorazione}

In inchiostro rosso il titolo e l'iniziale minore ad apertura del testo (f. 17r).

\section{Contenuto}

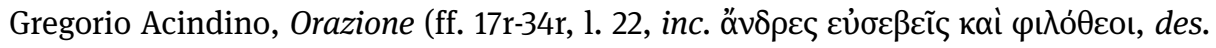

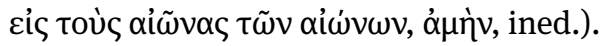

\section{III. ff. 35-98 (Costantinopoli, post 1344)}

Cart.; fil. ff. 37, 38, 40, 41, 43, 45, 47, 51, 53, 55, 56, 58, 61, 62, 64, 65, 66, 67, 68, 73, 75, 77, 79, 83, 84, 87, 91, 93 simile a arbalète $(\mathrm{M} / \mathrm{Tr} \mathrm{nr} .1342,1350) ; 4^{4}(38), 5^{8+2}(48), 6-11^{8}(96), 12^{4-2}(98)$; num. fasc. presente nel marg. inf. del recto del primo e nel marg. inf. int. del verso dell'ultimo foglio di ogni fascicolo, numerati da $\alpha^{\prime}$ a $\theta^{\prime} ; \mathrm{mm} 277 \times 207=25 / / 207 / / 43 \times 23$ // 5 // 141 // 5 // 29; a piena pagina, $11.34 / \mathrm{rr}$. 34, unità di rigatura $\mathrm{mm} 5$; rigatura tracciata a secco sul verso di ogni foglio, tipo S/L 20D1 (Muz 2-2/0/0/J) (f. 87).

\section{Scrittura}

L'unità è vergata da un solo copista: <Michele Balsamone (Mondrain 2006, 381; Mondrain 2007 [2008], 169)> (ff. 35v-98v, 1l. 21).

\section{Decorazione}

In inchiostro rosso il pinax al f. 35v, i titoli delle varie epistole e le iniziali minori al principio dei testi. Sempre in inchiostro rosso è vergata l'introduzione alle Refutationes al f. $91 \mathrm{v}$. 


\section{Interventi successivi alla copia}

Ad eccezione dell'annotazione al f. 40v, che deve essere attribuita a <Giovanni Cortasmeno>, tutti gli altri marginalia presenti nell'unità si devono al copista principale.

\section{Contenuto}

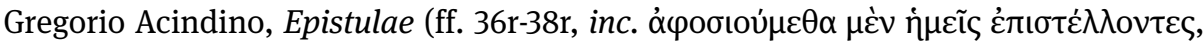

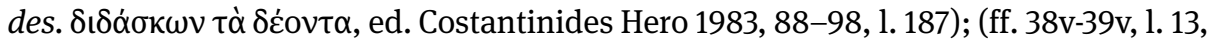

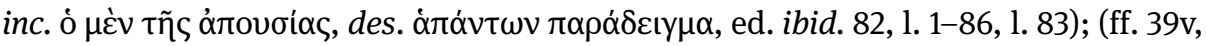

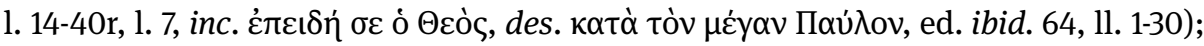

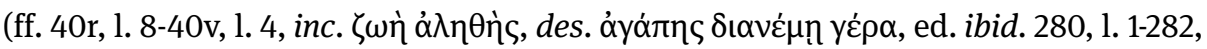

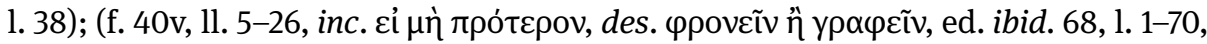

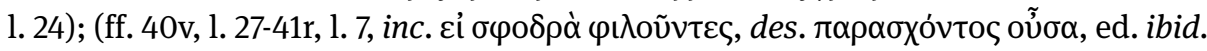

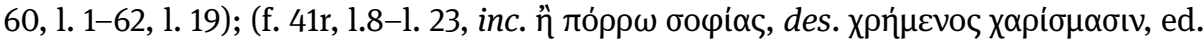

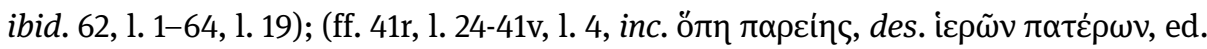

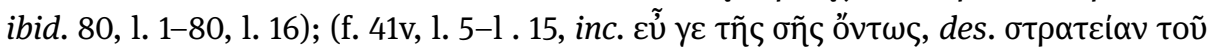

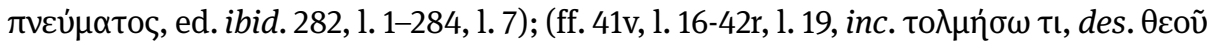

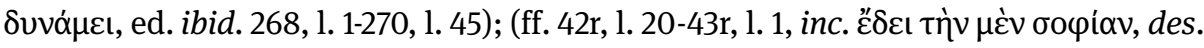

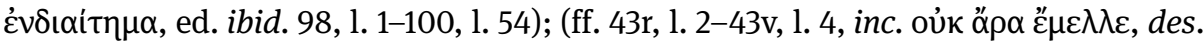

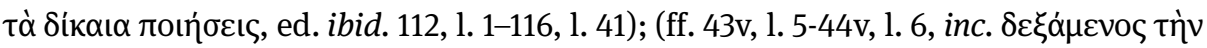

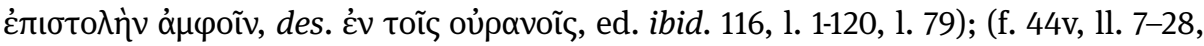

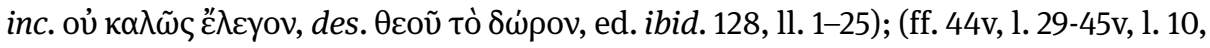
inc. Tò $\mu \varepsilon \dot{~ \varepsilon ́ ~ \varepsilon ́ \mu o i, ~ d e s . ~ \pi \alpha \lambda ı v \omega \delta i ́ \alpha v ~ o ̛ ̣ ~} \delta \omega v$, ed. ibid. 300, 1. 1-304, 1. 52); (ff. 45v, 1. 11-46r,

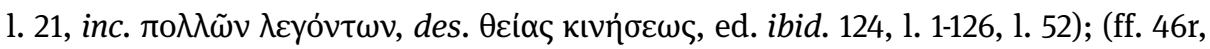

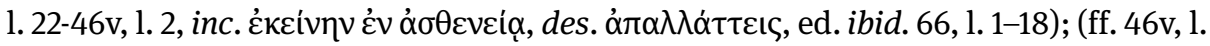

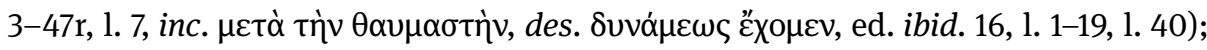

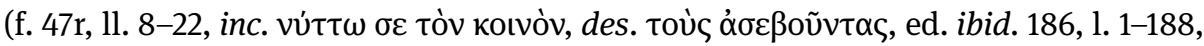

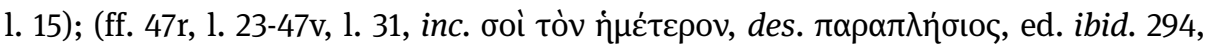

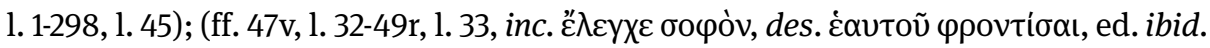

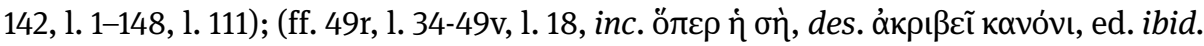

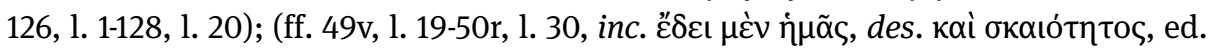

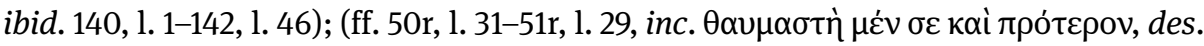

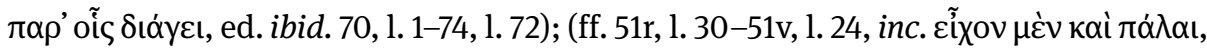

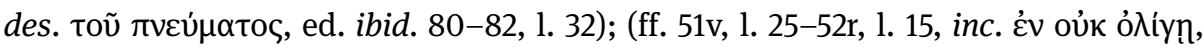

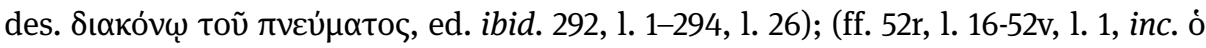

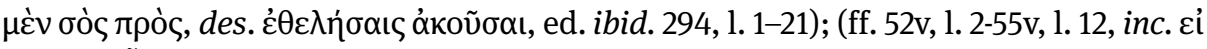

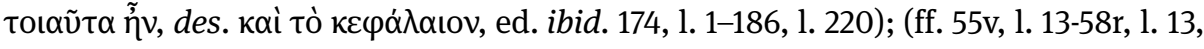

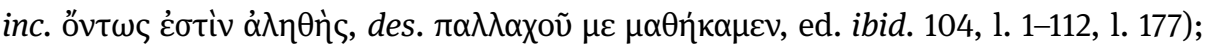

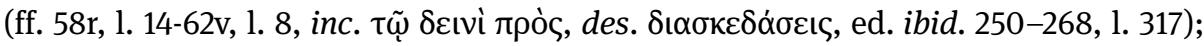




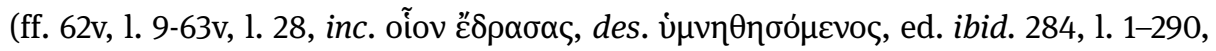

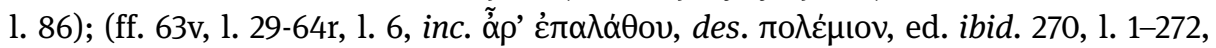

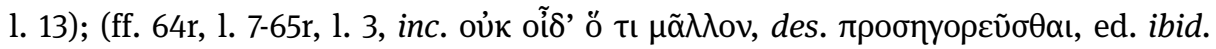

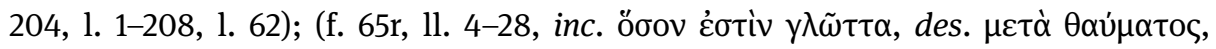

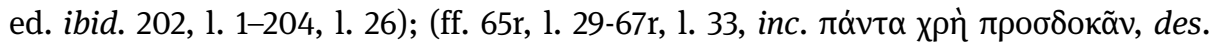

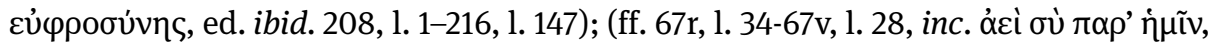

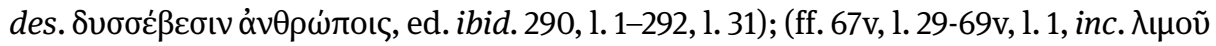

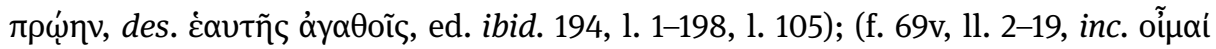

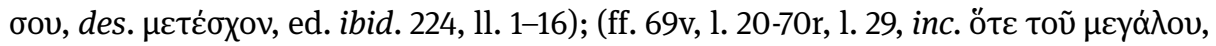

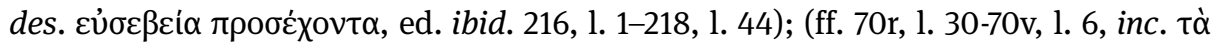

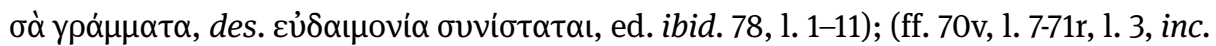

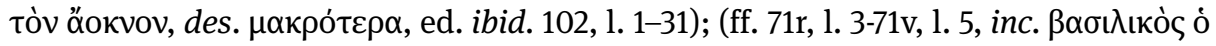

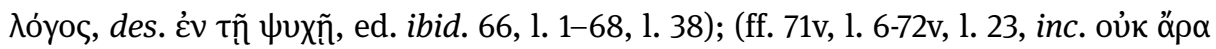

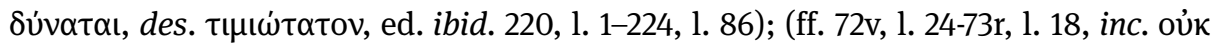

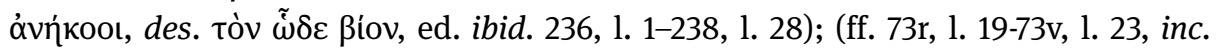

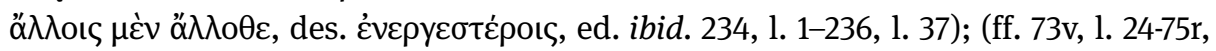

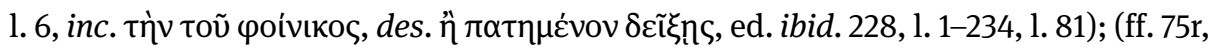

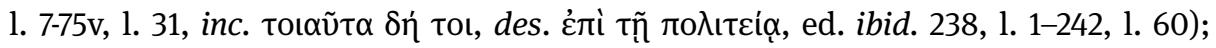

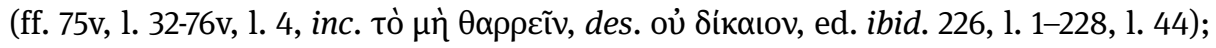

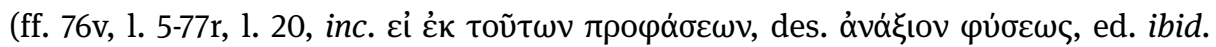

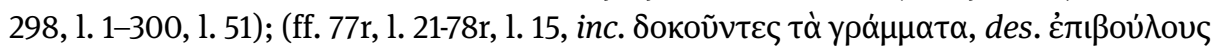

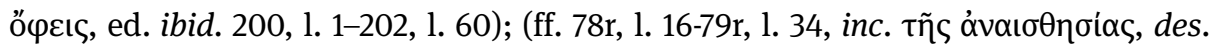

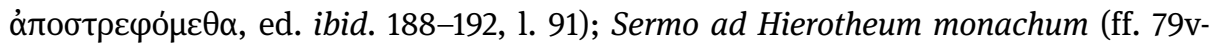

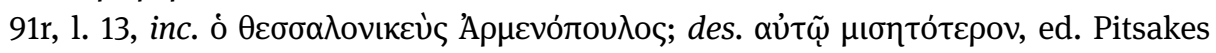

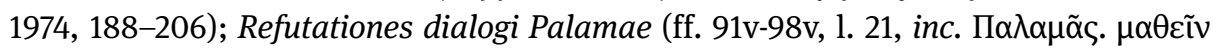

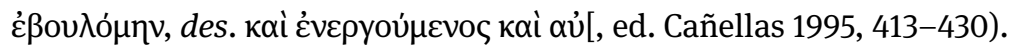

\section{IV. ff. 99-194 (Costantinopoli, XIV sec.)}

Cart.; fil. ff. 99, 102, 104, 105, 107, 111, 112, 113, 116, 117, 119, 121-125, 127, 133, 134, 137-140, 141, 143, 147, 149, 150, 153, 158, 160, 161, 162, 164, 165, 167, 170, 171, 175, 176, 177, 179, 180, 182, 184, 190, 192-194 simile a fruit (M/Tr nr. 4319, 1362); 13-24 (194); num. fasc. nel marg. inf. del verso dell'ultimo foglio di ogni fascicolo, numerati da $\alpha^{\prime}$ a ı $\beta^{\prime} ; \mathrm{mm} 275 \times 203=29$ // 209 // $40 \times 22$ // 146 // 36; a piena pagina, 1l. 30, unità di rigatura mm 6; rigatura assente (f. 131).

\section{Scrittura}

L'unità è vergata da un solo copista: <Anonimo G> (ff. 99r-194r). 


\section{Decorazione}

Rubriche in rosso carminio.

\section{Contenuto}

Procoro Cidone, De essentia et operatione libri VI, Liber I (ff. 99r-106v, 1. 26, inc. Toù

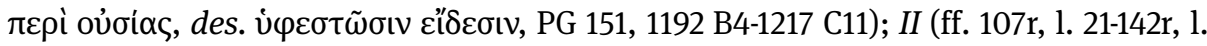

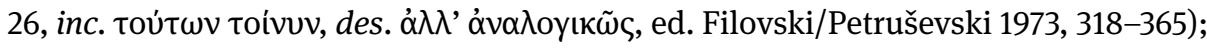

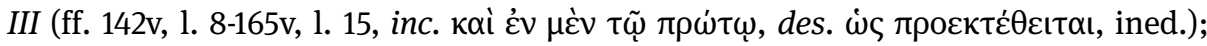

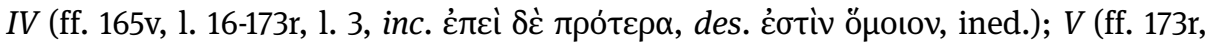

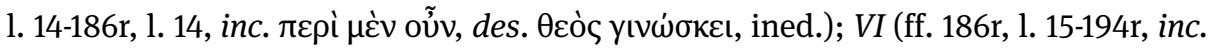

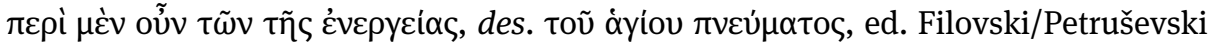
1976, 487-499).

\section{V. ff. 195-198 (Costantinopoli, XIV sec.)}

Cart.; fil. f. 197 simile a hache (M/Tr nr. 4681, 1358), f. 198 simile a fruit (M/Tr nr. 4319, 1362); 254 (198); num. fasc. assente; $\mathrm{mm} 274 \times 201=23$ // 216 // $35 \times 16$ // 170 // 23; a piena pagina, 11. 43, unità di rigatura $\mathrm{mm} 4$; rigatura assente (f. 196).

\section{Scrittura}

L'unità è opera di un solo copista: <Isacco Argiro (Mondrain 2007 [2008], 168; Bianconi 2008, 365)> (ff. 195r-198r).

\section{Decorazione}

Rubriche in inchiostro rosso.

\section{Contenuto}

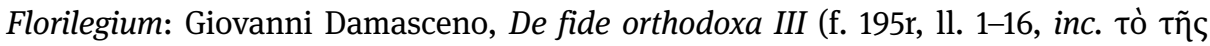

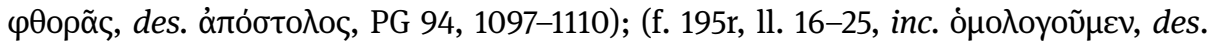

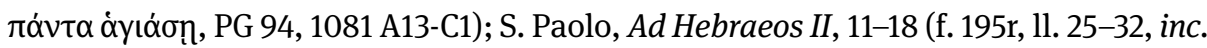

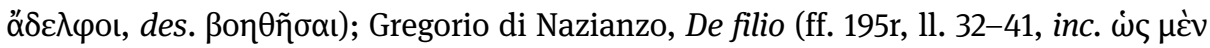

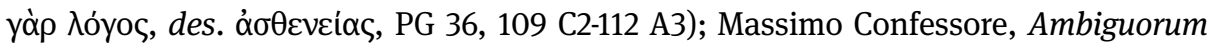




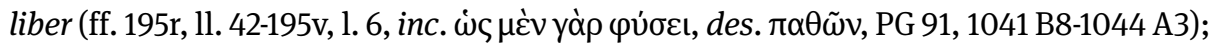

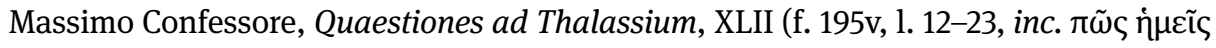

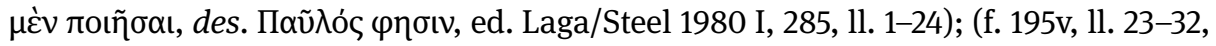

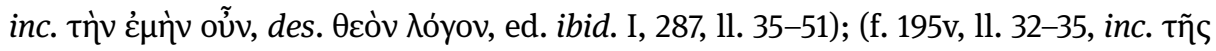

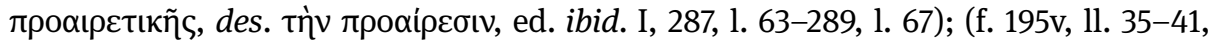

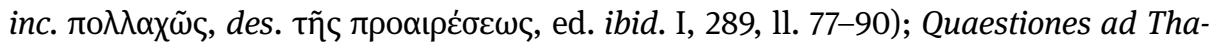

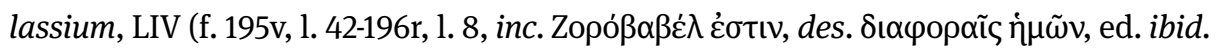

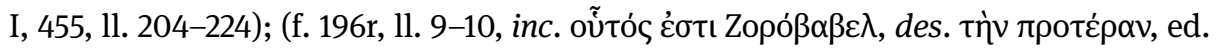

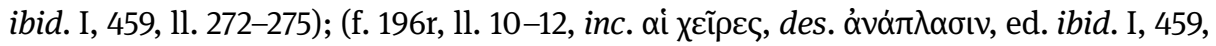

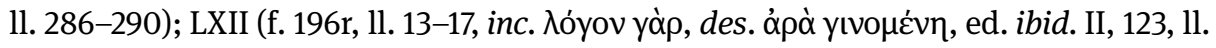

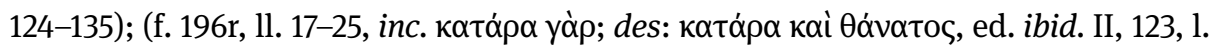

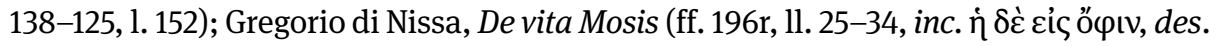

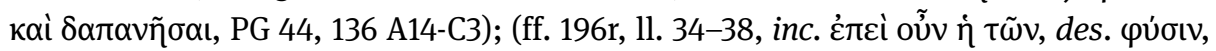
PG 44, 413 D10-416 A4); Giovanni Crisostomo, In epistulam ad Romanos (ff. 196r, 11.

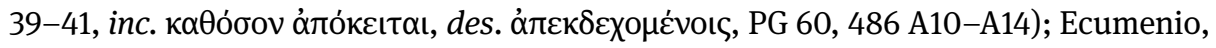

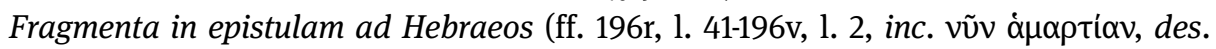

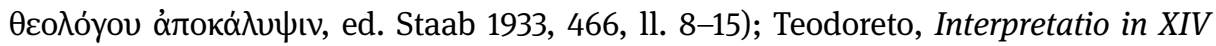

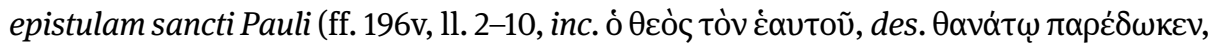
PG 82, 129 A1-7); Gennadio, Fragmenta in epistulam ad Romanos (f. 196v, 1l. 20-24, inc.

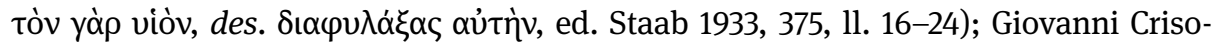

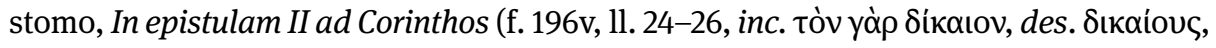
PG 61, 478 45-46); Didimo, Fragmenta in epistulam II ad Corinthos (f. 196v, 11. 27-29,

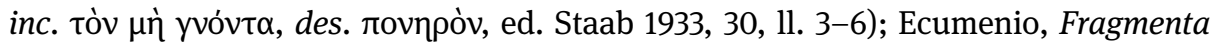

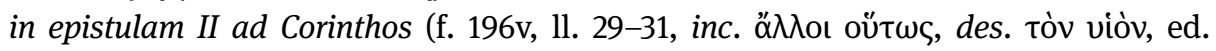
Staab 1933, 444, 1l. 16-19); Catenae in Novum Testamentum, Catena in epistulam ad

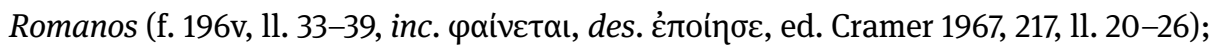
Demetrio Cidone, Liber sententiarum ex operibus S. Augustini (f. 196v, 1l. 40-44, inc.

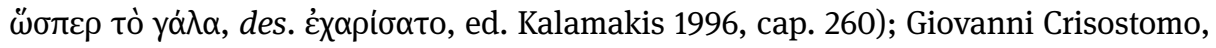

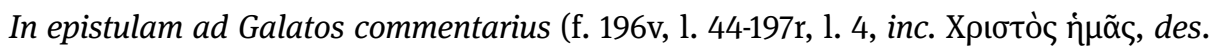

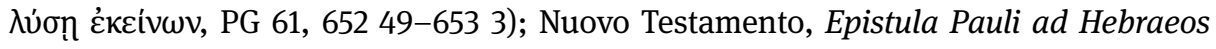

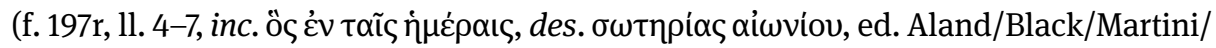

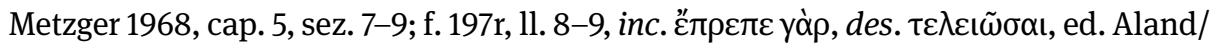
Black/Martini/Metzger 1968, cap. 2, sez. 10); Cirillo, Catena in epistulam ad Hebraeos

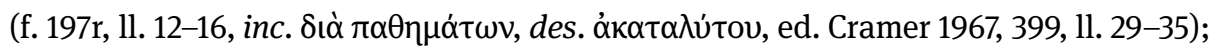

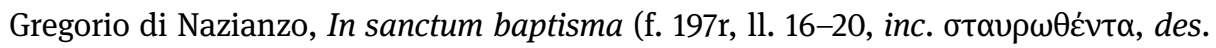

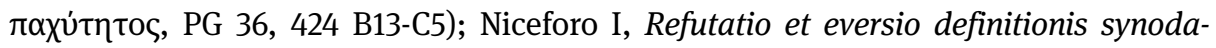

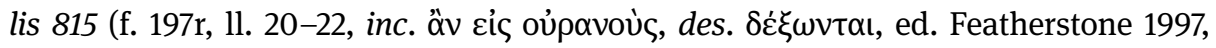
sez. 131, ll. 1-4); Gregorio di Nazianzo, In sanctum pascha (f. 197r, 1l. 22-25, inc. kồv

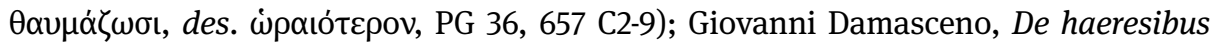

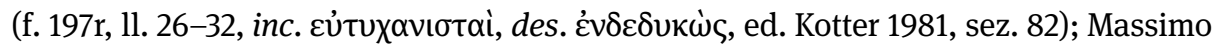
Confessore, Quaestiones ad Thalassium XXI (f. 197r, 1. 33-197v, 1. 25, inc. tí ćotìv, des.

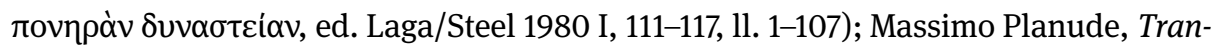




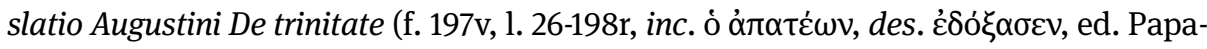
thomopoulos/Rigotti/Tsavare 1995 I, cap. 13, 11. 27-63); Teofilatto di Bulgaria, In Lucam

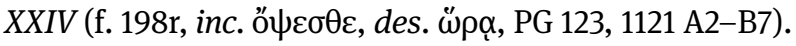

\section{VI. ff. 199-205 (Costantinopoli, XIV sec.)}

Cart.; fil. ff. 200, 201 simile a peson ou poids (M/Tr nr. 6791, 1348); $26^{8}(205)$; num. presente nel marg. inf. del recto del primo foglio del primo fascicolo, numerato $\alpha^{\prime} ; \mathrm{mm} 274 \times 202=34 / / 200 / /$ $44 \times 23$ // 67 // 13 // 64 // 35; due colonne, 1l. 33, unità di rigatura mm 4; rigatura assente (f. 202).

\section{Scrittura}

Nell'unità si rileva una solo mano: A (ff. 199r-204v), riferibile al XIV secolo; la parte finale di questa sezione si trova nel Marc. gr. Z. 582 (coll. 926) (Bianconi 2005, 110-112).

\section{Contenuto}

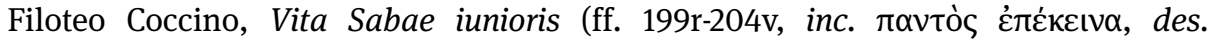

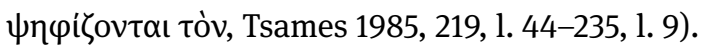

\section{Relazioni stemmatiche}

Il codice Marc. gr. Z. 155 (M) conserva cinquantuno lettere di Gregorio Acindino, alcune delle quali sono trasmesse anche nei codici Monac. gr. 233, Vatt. grr. 1086 e 1122. Come in questi ultimi, anche nel Marciano le lettere sono raccolte in modo disordinato (Costantinides Hero 1983, XXXVII).

\section{Storia del codice}

Il codice, appartenuto alla collezione libraria di Bessarione, figura con il numero 172 nell'inventario del 1468, stilato in occasione della donazione della biblioteca da parte del cardinale alla basilica di San Marco a Venezia (Labowsky 1979, 162). 


\section{Bibliografia}

\section{Cataloghi}

Mioni (1981) 225-228.

\section{Edizioni}

Aland/Black/Martini/Metzger (1968); Cañellas (1995); Cramer (1967); Featherstone (1997); Filovski/ Petruševski (1973); Filovski/Petruševski (1976); Kalamakis (1996); Kotter (1981); Laga/Steel (1980); PG 36; PG 44; PG 60; PG 61; PG 82; PG 91; PG 94; PG 123; PG 151; Papathomopoulos/ Rigotti/Tsavare (1995) I; Pitsakes (1974); Staab (1933) 466; Tsames (1985).

\section{Codicologia e paleografia}

Bianconi (2005) 110-112; Bianconi (2008) 365-366; De Gregorio (2014) 189 n. 31; Labowsky (1979) 162; Mercati (1931) 2, 10, 40 n. 3, 485-488; Mondrain (2006) 381; Mondrain (2007) [2008] 168-169; Stefec (2013) 49.

\section{Riproduzioni}

Bianconi (2008) tav. 18. 